\title{
Paper
}

\section{Mobile Robot for Environmental Measurement in Greenhouse}

\author{
KENICHI IIDA*† \\ Member, \\ HikARU Kumamoto \\ Member \\ Shigeto Nakamura ${ }^{\dagger}$ Member, \\ ETSUKO UEDA ${ }^{\S}$ \\ Non-member
}

(Received September 29, 2019, revised January 08, 2020)

\begin{abstract}
The environmental information in a greenhouse is one of the essential items in precision agriculture for improving the productivity of crops. In many cases of environmental measurement in a greenhouse, a fixed sensor can take measurements near the installation location. However, to estimate the distribution of environmental information in the entire greenhouse, it is necessary to install many sensors, which increases the cost. Therefore, in this study, to acquire more detailed environmental information, we propose a system to measure the environmental information in a greenhouse by using a mobile robot with Robot Operating System (ROS). The proposed mobile robot consists of a $\mathrm{CO}_{2}$ sensor, temperature sensor, humidity sensor, and Laser Range Finder (LRF) for environmental sensing. In this paper, we confirmed the usefulness of the proposed system from a verification experiment using a prototype mobile robot..
\end{abstract}

Keywords: Agricultural support, Environmental measurement, Mobile robot, ROS,

\section{Introduction}

For sustainable agricultural growth, efficient and stable agricultural management [1] is required. Therefore, the Ministry of Agriculture, Forestry and Fisheries promotes precision agriculture based on environmental information on farms to control the production of crops without depending on experience and intuition, and the smart-agriculture using advanced technologies such as Artificial Intelligence (AI) [2], Internet of Things (IoT) [3] [6], and robots [7] [11]. There have been many studies [12] [16] about environmental measurement so that crops can be cultivated based on quantitative environmental information. Many studies acquired environmental data from fixed environmental sensors on farmland. For example, Suyama et al. proposed a method of environmental sensing in a plastic greenhouse by using a sensor network [17], and Fukatsu et al. developed field servers for a field monitoring system [18].

However, the measuring range of fixed sensors is restricted. Thus, it is difficult to obtain detailed information about the entire greenhouse. Consequently, in the case of a large-scale greenhouse, many sensors are required, and the cost increases.

In this study, we propose a detailed environmental measurement system by automatic patrol using a mobile robot equipped with an environmental sensor unit, assuming a large-scale greenhouse.

\footnotetext{
* Corresponding: iida@ctrl.nara-k.ac.jp

$\dagger$ National Institute of Technology, Nara College

22 Yata, Yamatokoriyama, Nara, Japan 639-1080

$\$$ Nara Institute of Science and Technology

8916-5 Takayama-cho, Ikoma, Nara, Japan 630-0192

$\S$ Osaka Institute of Technology

1-45 Chayamachi, Kita-ku, Osaka, Japan 530-8568
}

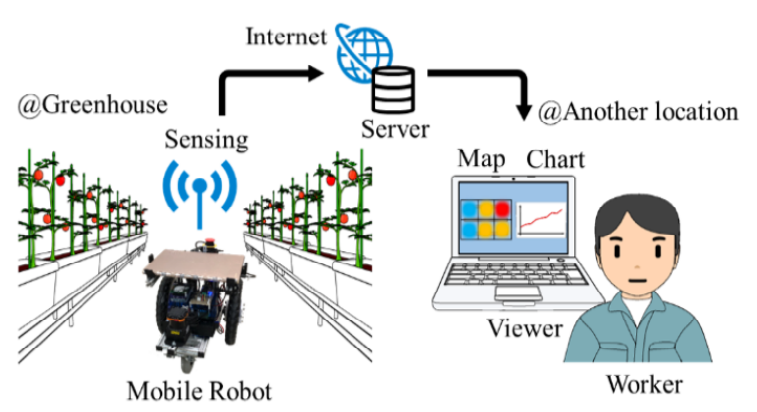

Figure 1: Outline of proposed system.

\section{Proposed System}

2.1 System Configuration Figure 1 shows the outline of the proposed system. The mobile robot acquires the detailed environmental information by automatic patrol between the benches in the elevated cultivation greenhouse. The obtained environmental information can be used by farmers as an environment map in the greenhouse. Fig. 2 and Fig. 3 show an overview of the mobile robot and the system configuration, respectively. The mobile robot has two wheels and a caster attached to the front. An environmental sensor unit, as shown in Fig. 4, is equipped with a $\mathrm{CO}_{2}$ sensor, temperature sensor, and humidity sensor. The robot drive unit has DC motors, motor drivers, and rotary encoders on the left and right wheels for driving and reading the rotation velocity, as shown in Fig. 5. As the mobile robot patrols the greenhouse, a LRF acquires the layout and obstacle information. The mobile robot runs on ROS, which is a framework in robot development [19] [20].

Table $1 \sim$ Table 4 list the specifications of the mobile robot, environmental sensors, motor with the encoder, and LRF, respectively. 


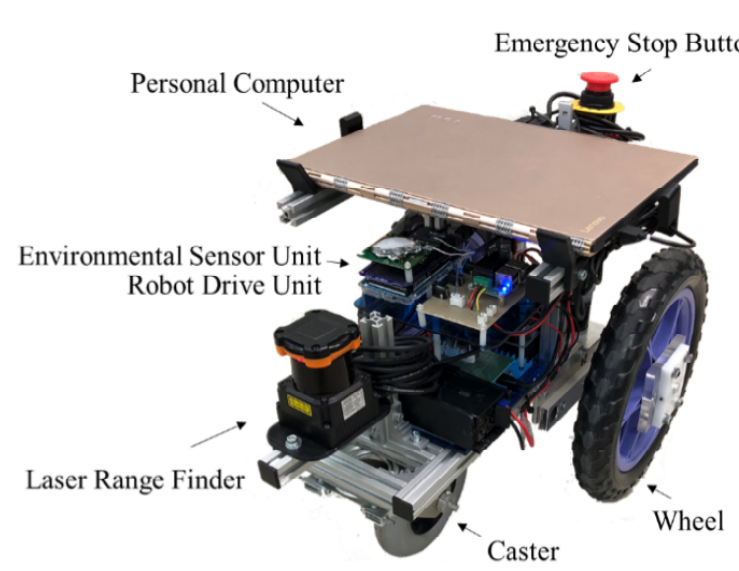

Figure 2: Overview of mobile robot.

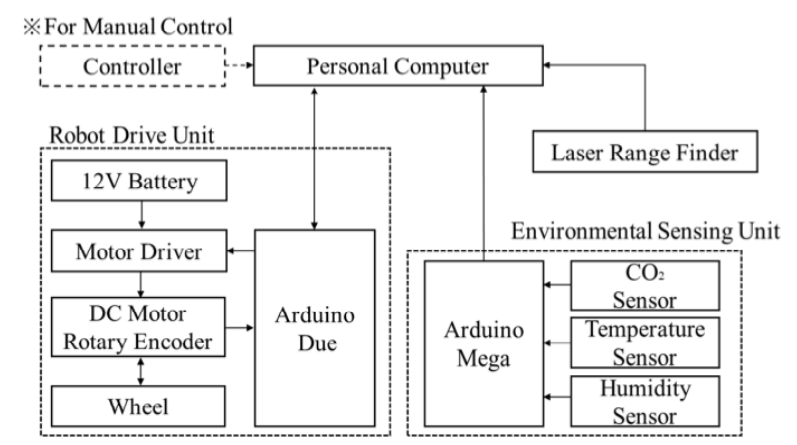

Figure 3: System configuration.

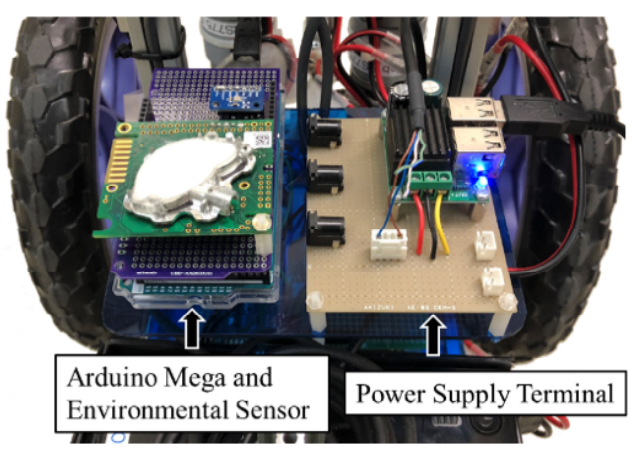

Figure 4: Overview of environmental sensor unit.

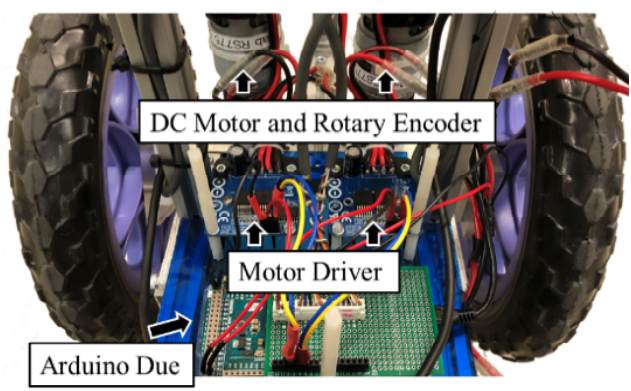

Figure 5: Overview of robot drive unit.

2.2 Patrolling Mobile Robot using ROS Automatic patrol using the proposed system is conducted by the following two procedures.
Table 1: Specifications for mobile robot.

\begin{tabular}{lc}
\hline Size $(\mathrm{W} \times \mathrm{D} \times \mathrm{H})[\mathrm{mm}]$ & $300 \times 500 \times 450$ \\
\hline Tread Width $[\mathrm{mm}]$ & 292 \\
Wheel Diameter $[\mathrm{mm}]$ & 294 \\
Weight $[\mathrm{kg}]$ & 7.8 \\
Payload $[\mathrm{kg}]$ & 2.2 \\
Maximum Speed $[\mathrm{km} / \mathrm{h}]$ & 7.2 \\
\hline
\end{tabular}

Table 2: Specifications for sensors.

\begin{tabular}{lcc}
\hline- & Range & Accuracy \\
\hline $\mathrm{CO}_{2}[\mathrm{ppm}]$ & $0 \sim 2000$ & \pm 30 \\
Temperature $\left[{ }^{\circ} \mathrm{C}\right]$ & $-40 \sim+85$ & \pm 1 \\
Humidity $[\%]$ & $0 \sim 100$ & \pm 3 \\
\hline
\end{tabular}

Table 3: Specifications for motors with encoders.

\begin{tabular}{lc}
\hline Item & Specification \\
\hline Motor Rated Voltage [V] & 18 \\
Motor Rated Revolution [rpm] (No Load) & $219(250)$ \\
Encoder Resolution [ppr] & 360 \\
\hline
\end{tabular}

Table 4: Specifications for LRF.

\begin{tabular}{lc}
\hline Item & Specification \\
\hline Range & 0.1 to $30[\mathrm{~m}], 270\left[^{\circ}\right]$ \\
\hline Accuracy & 0.1 to $10[\mathrm{~m}]: \pm 30[\mathrm{~mm}]$ \\
& 10 to $30[\mathrm{~m}]: \pm 50[\mathrm{~mm}]$ \\
\hline Angular Resolution & $0.25\left[^{\circ}\right]\left(270\left[{ }^{\circ}\right] / 1080[\mathrm{steps}]\right)$ \\
\hline
\end{tabular}

Procedure 1: Creation of the map. The operator manually operates the mobile robot to acquire the layout information in the greenhouse measured by the LRF and the odometry of the mobile robot. The obtained information creates a map necessary for the mobile robot to travel autonomously using the "slam_gmapping stack" of ROS.

Procedure 2: Setting of the route of the patrol. Based on the map acquired in Procedure 1, the operator sets multiple target points on the route for measuring environmental information. The mobile robot patrols by repeating the autonomous traveling information to each set target point. Then, the mobile robot can move autonomously by adopting the "navigation stack" of ROS.

Figure 6 shows the configuration of the ROS packages for the proposed patrol system. The function of the ROS packages is described below. First, "navigation" consists of three packages: "move_base", "map_server", and "amcl" (Adaptive Monte Carlo Localization). "move_base" is a framework of autonomous movement and consists of "global_planner", "local_planner", "global_costmap", and "local_costmap" for the management of obstacles, "recovery_behaviors" for the robot stack status to move the robot to the target point on the created map. "map_server" provides map information to "move_base" and "amcl". Then, 
"amcl" performs localization with scan data from the LRF and the odometry of the robot.

The proposed system is introduced with "urg_node", which is a driver for acquiring scan data from the LRF; "tf" to transform the coordinate frames of the map, mobile robot, and the LRF; and "rosserial", which is a protocol for serial devices such as the Arduino. The proposed system introduces "odom_node" to estimate the amount of movement of the mobile robot.

Subsequently, "odom_node" provides the odometry of the mobile robot to "move_base" and "amcl" from the kinematics of the two-wheeled robot, as shown in Fig. 7. In this figure, $r$ is the turning radius, $v$ is the straight traveling velocity, $w$ is the angular velocity, $d$ is half the tread width, and $v_{r}$ and $v_{l}$ are the right and left wheel velocities, respectively. The geometric relationships between every parameter in Fig. 7 are shown by Eq. 1 and Eq. 2.

$$
\begin{aligned}
& r=v / w \\
& \left.\begin{array}{l}
v_{r}=(r+d) w \\
v_{l}=(r-d) w
\end{array}\right\}
\end{aligned}
$$

As the amount of movement of the robot, the straight traveling velocity $v$ and angular velocity $w$ are estimated by Eq. 3.

$$
\left.\begin{array}{c}
v=\left(v_{r}+v_{l}\right) / 2 \\
w=\left(v_{r}-v_{r}\right) / 2 d
\end{array}\right\}
$$

The setting of the target points using "navigation" is generally performed by the Graphical User Interface (GUI) using visualization tool "rviz". However, the GUI in visualization tool "rviz" cannot set subgoals en route to the final destination. It is possible to set a subgoal en route to the final destination by using the newly developed "goal_node" in the proposed system. Thus, it is possible to design the arbitrary route of the patrol.

\subsection{Presentation of Environmental Information}

The measured environmental information by patrolling using a mobile robot is the data on the coordinates of each measurement point. It is difficult for the farmer to understand the conditions in the entire greenhouse. Thus, to present the case in the entire greenhouse to the farmer more effectively, we created an environmental map that overlays the measured environmental information on the greenhouse layout. Fig. 8 shows the visualization flow of the environmental information. The environmental map is generated by executing a python script with the $2 \mathrm{D}$ occupancy grid map and integrating environmental information and traveling locus in the ROS. The environmental data are shown in the greenhouse layout. Hence, the farmer can easily understand the conditions in the entire greenhouse.

\section{Evaluation Experiment}

To verify the usefulness of the proposed system, we conducted an environmental measurement experiment by patrolling an actual elevated cultivation greenhouse using the mobile robot, as shown in Fig. 9. Fig. 10 shows the layout

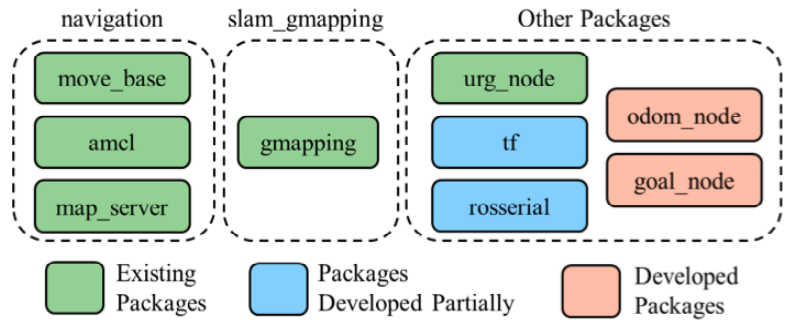

Figure 6: Configuration of ROS packages.

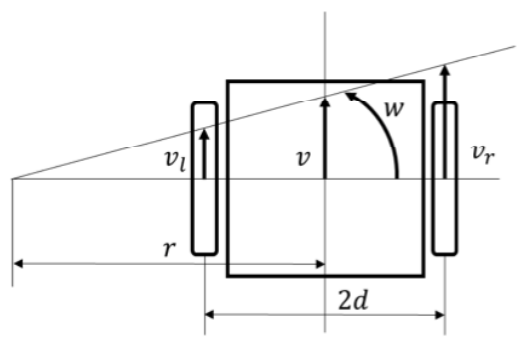

Figure 7: Kinematics of two-wheel robot.

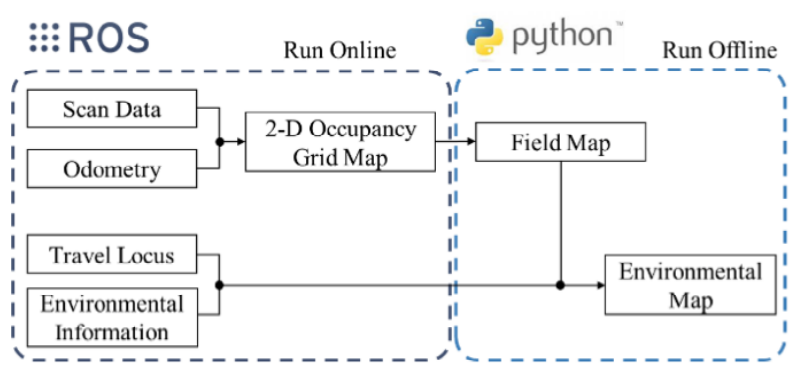

Figure 8: Visualization flow of environmental information.

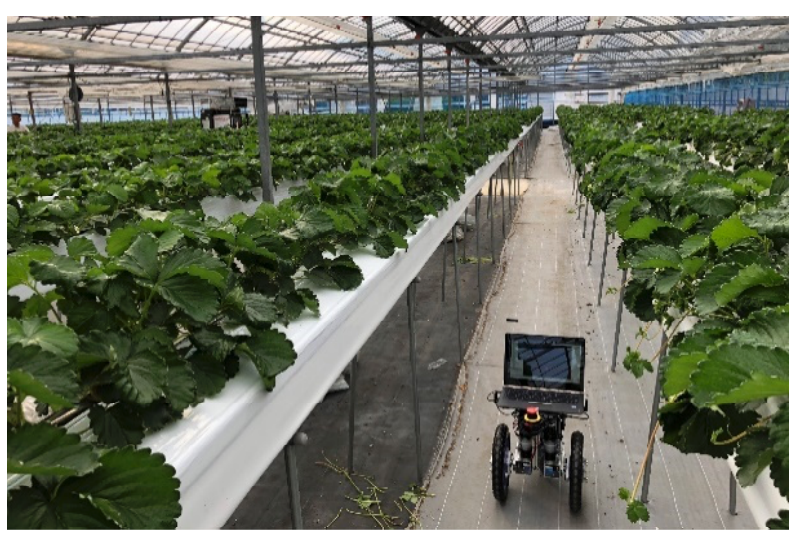

Figure 9: Overview of greenhouse.

of the greenhouse. In the figure, (1)-(5) are the target points, and the blue arrows denote the traveling route.

The mobile robot travels at approximately $0.17 \mathrm{~m} / \mathrm{s}$ and takes environmental measurements every second. Therefore, the mobile robot can obtain data that are approximately equal to the environmental data measured by installing approximately 58 sensors at $10 \mathrm{~m}$ intervals. As shown in Fig. 9, the mobile robot traveled and acquired the layout in the entire greenhouse.

Figure 11 shows the 2D occupancy grid map generated 


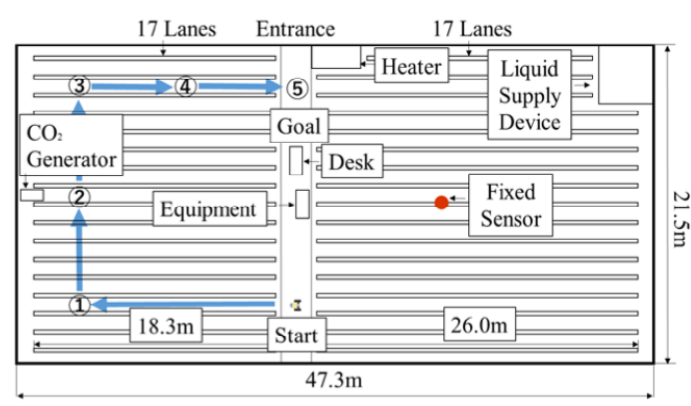

Figure 10: Condition of greenhouse.

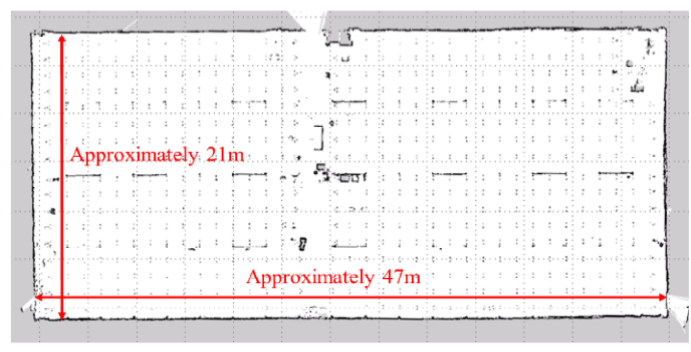

Figure 11: Generated map of greenhouse.

using "slam_gmapping". This confirmed that the actual layout of the greenhouse had been reproduced. Fig. 12 shows the trajectory of the mobile robot and the humidity with the measured on the travel locus. As shown in the figure, the environmental measurement points are indicated on the actual layout of the greenhouse, and it is possible to confirm the distribution of the humidity.

In addition, in this experiment, about 500 environmental measurement points were acquired by patrolling. Hence, it was confirmed that the detailed measurement of environmental information is possible. Furthermore, it is possible to adjust the measurement points, e.g., arbitrary positions of the measurement points, by the patrolling route, the number of measurement points by the robot velocity, or the measurement sampling rate. Fig. 13 shows interpolation maps by the inverse distance weight (IDW) calculated using Eq. 4 and Eq. 5 from the obtained environmental data.

$$
\begin{aligned}
& u(x)=\frac{a \sum_{i=0}^{N} w_{i}(x) u\left(x_{i}\right)}{\sum_{j=0}^{N} w_{j}(x)} \\
& w_{i}(x)=\frac{1}{d\left(x, x_{i}\right)^{p}}
\end{aligned}
$$

where $x$ is the estimated point, $\mu(x)$ and $w_{i}(x)$ are each characteristic value, $\mathrm{d}\left(x, x_{i}\right)$ is the distance between two points, and $p$ is the distance index.

As shown in the Fig. 13, the measured point group data is converted to planar information by interpolation. Hence, the farmer can visually confirm the distribution of the detailed environmental information of the left side of the greenhouse. In addition, more precise interpolation is possible by setting the traveling route in detail. By contrast, the distribution of the detailed environmental information on the right side of the greenhouse cannot be confirmed because only fixed sensor data were used. Therefore, the pro-

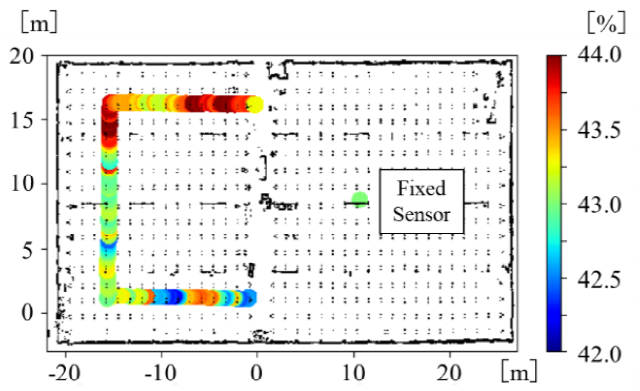

Figure 12: Trajectory of robot and measurement data of humidity.

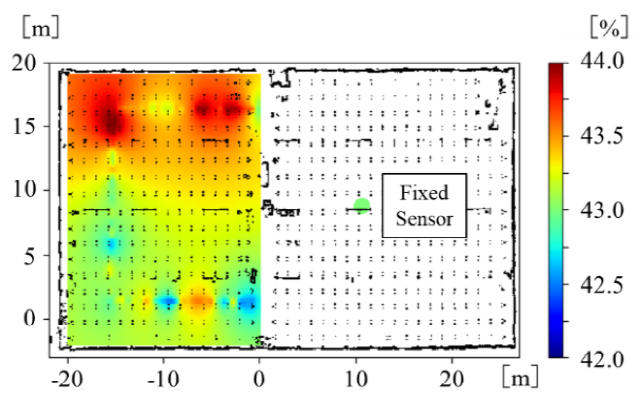

Figure 13: Interpolation map using IDW of humidity.

posed system by the mobile robot can acquire more detailed environmental data in the greenhouse.

Next, we conducted a patrolling experiment in the greenhouse with a mobile robot using ROS. The patrolling experiment was performed every hour, and environmental data were measured every second. Some results of the experiments are shown in Fig. 14 and Fig. 15. From Fig. 14(a), Fig. 14(b) and Fig. 14(c), it is confirmed that the mobile robot travels along the setting route every time and measures the environmental data. Furthermore, by interpolation with IDW, environmental maps of the entire greenhouse can be generated, such as Fig. 15(a), Fig. 15(b), and Fig. 15(c). Hence, farmers can easily understand differences in the environmental data owing to the measured time in the greenhouse.

\section{Conclusions}

In this study, we proposed a detailed environmental measurement system in a greenhouse by patrolling using a mobile robot. Experimental results in the greenhouse demonstrated that the mobile robot could patrol designated routes while measuring environmental information. A mobile robot equipped with a sensor unit acquired many environmental measurement data items. Hence, the usefulness of the proposed system was confirmed. Although it is desirable to patrol all lanes for $24 \mathrm{~h}$ a day ideally, the time and cost depend on the scale of the greenhouse. Therefore, it is necessary to consider the optimum measurement frequency and area.

The proposed method can be applied to measure the full environmental distribution in factories and offices. We are planning improvements to the proposed system so that farmers can more easily use the measured environmental in- 


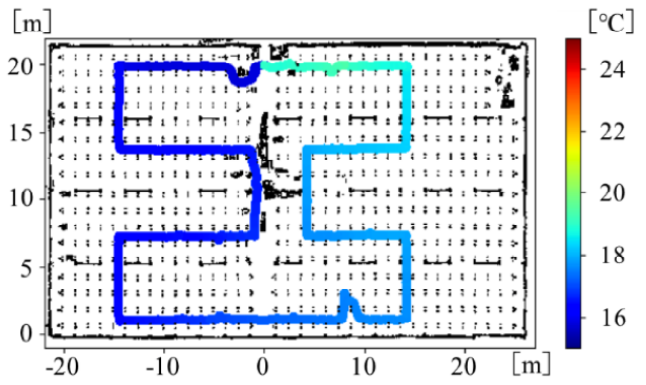

(a) 9:00

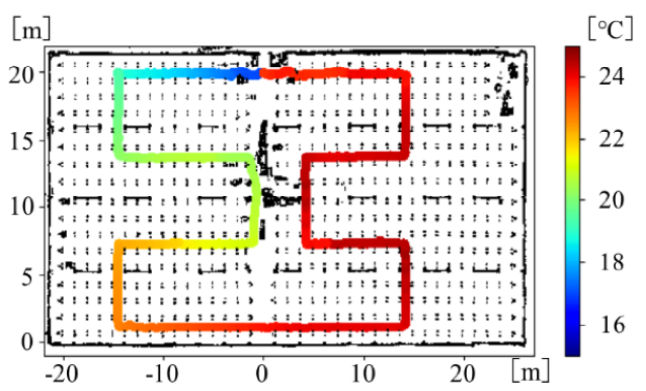

(b) 12:00

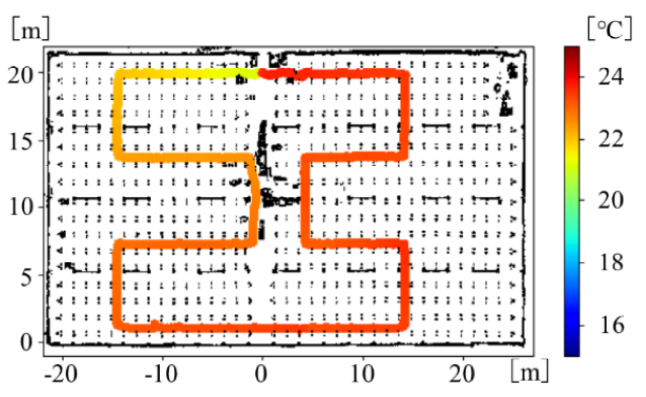

(c) $15: 00$

Figure 14: Trajectory of robot and measurement data of temperature.

formation. These improvements include IoT cloud service and completely automatic sensing.

\section{References}

[1] "Annual Report on Food, Agriculture and Rural Areas trends in Japan FY 2017", The Ministry of Agriculture, Forestry and Fisheries of Japan, pp.1, 2017.

[2] Shumpei Uegaki, Hidekazu Araki, Ryo Toshima, Makoto Shinzaki, Daisuke Ueta, and Ryuji Yamazaki, "TomatoHarvesting Robot using AI for Environment Recognition", Panasonic Technical Journal, Vol.64, No.1, pp.54-59, 2018.

[3] Karim Foughali, Karim Fathallah and Ali Frihida, "Using Cloud IOT for disease prevention in precision agriculture", Procedia Computer Science, Vol.130, pp.575-582, 2018. DOI: $10.1016 /$ j.procs.2018.04.106

[4] I. Mohanraj, Kirthika Ashokumar and J. Naren, "Field Monitoring and Automation using IOT in Agriculture Domain", Procedia Computer Science, Vol.93, pp.931-939, 2016. DOI: 10.1016/j.procs.2016.07.275

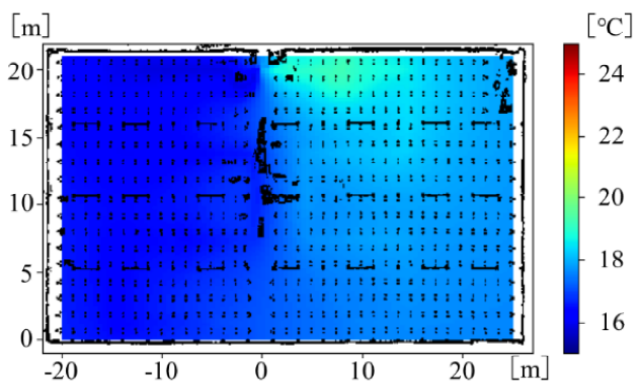

(a) $9: 00$

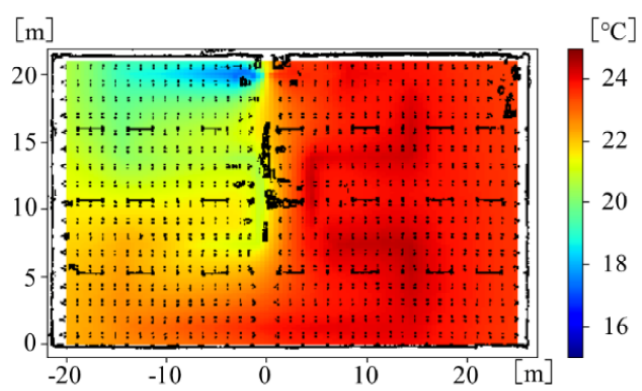

(b) $12: 00$

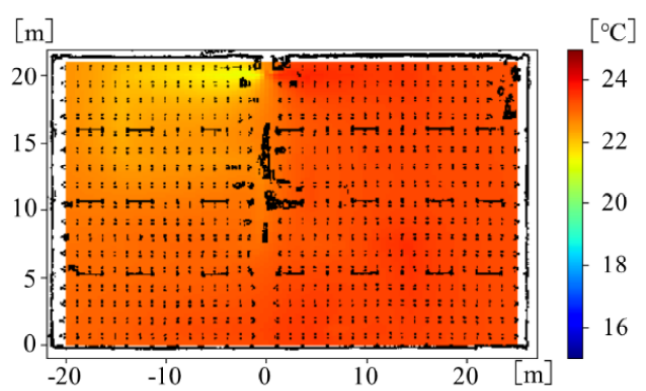

(c) $15: 00$

Figure 15: Environmental map using IDW of temperature.

[5] Mustafa Alper Akkasa and Radosveta Sokullu, "An IoTbased greenhouse monitoring system with Micaz motes", Procedia Computer Science, Vol.113, pp.603-608, 2017. DOI: 10.1016/j.procs.2017.08.300

[6] Foughali Karim, Fathalah Karim and Ali frihida, "Monitoring system using web of things in precision agriculture", Procedia Computer Science, Vol.110, pp.402-409, 2017. DOI: 10.1016/j.procs.2017.06.083

[7] Juha Backman, Pyry Piirainen and Timo Oksanen, "Smooth turning path generation for agricultural vehicles in headlands", Biosystems Engineering, Vol.139, pp.76-86, 2015. DOI: 10.1016/j.biosystemseng.2015.08.005

[8] Josse De Baerdemaker, "Precision Agriculture Technology and Robotics for Good Agricultural Practices", IFAC Proceedings Volumes, Vol.46, No.4, pp.1-4, 2013. DOI: 10.3182/20130327-3-JP-3017.00003

[9] Philip J.Sammons, Tomonari Furukawa and Andrew Bulgin, "Autonomous Pesticide Spraying Robot for use in a Greenhouse", pp.1-9, 2005.

[10] Gilad Gat, Samuel Gan-mor and Amir Degani, "Stable and robust vehicle steering control using an over- 
head guide in greenhouse tasks", Computers and Electronics in Agriculture, Vol.121, pp.234-244, 2016. DOI: 10.1016/j.compag.2015.12.019

[11] Simon Janos, Goran Martinović and István Matijevics, "WSN Implementation in the Greenhouse Environment Using Mobile Measuring Station", International Journal of Electrical and Computer Engineering Systems, Vol.1, No.1, pp.37-44, 2010.

[12] I.-Chang Yang, Kuang-Wen Hsieh, Chao-Yin Tsai, Yu-I. Huang, Yu-Liang Chen and Suming Chen, "Development of an automation system for greenhouse seeding production management using radio-frequency-identification and local remote sensing techniques", Engineering in Agriculture, Environment and Food, Vol.7, No.1, pp.52-58, 2014. DOI: 10.1016/j.eaef.2013.12.009

[13] Javier López-Martínez, José L.Blanco-Claraco, José PérezAlonso and Ángel J.Callejón-Ferre, "Distributed network for measuring climatic parameters in heterogeneous environments: Application in a greenhouse", Computers and Electronics in Agriculture, Vol.145, pp.105-121, 2018. DOI: 10.1016/j.compag.2017.12.028

[14] B. Marchial, S. Zanonia and M. Pasetti, "Industrial Symbiosis for Greener Horticulture Practices: The $\mathrm{CO} 2$ Enrichment from Energy Intensive Industrial Processes", Procedia CIRP, Vol.69, pp.562-567, 2018. DOI: 10.1016/j.procir.2017.11.117

[15] Prashant Singh Chauhan, Anil Kumar and Chayut Nuntadusit, "Heat transfer analysis of PV integrated modified greenhouse dryer", Renewable Energy, Vol.121, pp.53-65, 2018. DOI: 10.1016/j.renene.2018.01.017

[16] J. del Sagrado, J. A. Sánchez, F. Rodríguez and M. Berenguel, "Bayesian networks for greenhouse temperature control", Journal of Applied Logic, Vol.17, pp.25-35, 2016. DOI: $10.1016 /$ j.jal.2015.09.006

[17] Takayuki Suyama, Futoshi Naya, and Yutaka Yanagisawa, "Environmental Sensing in a Plastic Greenhouse by Sensor Network", DICOMO 2013, pp.938-944, 2013.

[18] Tokihiro Fukatsu and Masayuki Hirafuji, "Development of Field Servers for a Field Monitoring System", Agricultural Information Research, Vol.12, No.1, pp.1-12, 2003. DOI: 10.3173/AIR.12.1

[19] Yoshitaka Hara, "Autonomous navigation with ROS", Journal of the Robotics Society of Japan, Vol.35, No.4, pp.286290, 2017. DOI: $10.7210 /$ jrsj.35.286

[20] Atsushi Watanabe, "Development of a ROS-based Oil Plant Inspection Mobile Robot System -Making Decision between ROS Ecosystem's and From-Scratch Packages-", Journal of the Robotics Society of Japan, Vol.35, No.4, pp.291-294, 2017. DOI: $10.7210 /$ jrsj.35.291

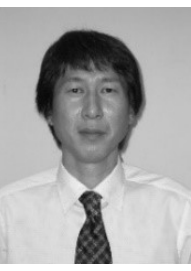

Kenichi Iida (Member) received the doctoral degree of engineering from Tokushima University in 1997. Currently, he is a professor in the Department of Control Engineering at the National Institute of Technology, Nara College. His current research activity is the intelligent control system of mobile robots and the application of the control system. He is a member of IEEJ, RSJ, SICE, JSAM, and IIAE.

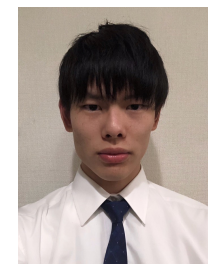

Hikaru Kumamoto (Member) graduated from the Faculty of Advanced Engineering, National Institute of Technology, Nara College, in 2019, and is presently a student at Nara Institute of Science and Technology. $\mathrm{He}$ is mainly engaged in research on control systems using mobile robots. He is a member of IEEJ and IIAE.

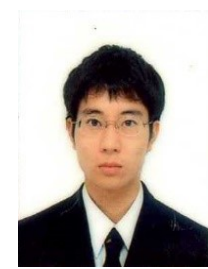

Shigeto Nakamura (Member) received a $\mathrm{Ph} . \mathrm{D}$. degree in engineering from Hokkaido University in 2011. He is an associate professor in the Department of Control Engineering at National Institute of Tech., Nara College. His research field is fluid mechanics. He is a member of JSME, JSFM, and IIAE.

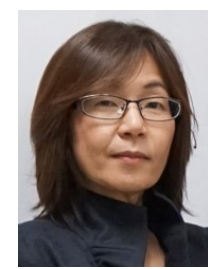

Ueda Etsuko (Non-member) received a Ph.D. degree in engineering from Nara Institute of Science and Technology in 2003. She is presently a professor in the faculty of Robotics \& Design at Osaka Institute of Technology. Her research field is human motion analysis and modeling. She is a member of IEEE, JSME, and RSJ. 